\title{
Originalarbeiten
}

\section{Bestimmung von Redoxzonen in einem mineralölbelasteten Grundwasserleiter}

\author{
Rudolf Huth*, Rainer Hartmann, Michaela Kiesel, Wilhelm Pyka und Annette Stallauer \\ Fachhochschule Weihenstephan, Fachbereich Umweltsicherung, Steingruberstrasse 2, D-91746 Weidenbach
}

* Korrespondenzautor (rudolf.huth@fh-weihenstephan.de)

$$
\text { DOI: http://dx.doi.org/10.1065/uwsł2004.08.084 }
$$

\section{Zusammenfassung}

Ziel und Absicht. Ziel dieser Untersuchung ist es, in einem Grundwasserleiter mit einer Mineralölkohlenwasserstoff-Verunreinigung die Ausbildung der folgenden charakteristischen Redoxzonierung in der Schadstofffahne der Kontamination zu ermitteln und grafisch darzustellen: Methanogene Bedingungen, Sulfatreduktion, Eisen(III)-Reduktion, Mangan(IV)-Reduktion, Nitratreduktion, aerobe Bedingungen. Damit sollen Hinweise auf Art und Ausmaß mikrobiologischer Abbauprozesse schnell und einfach erhalten werden, die im Zuge der Altlastenbearbeitung durch Einsatz von Natural Attenuation (NA) essentiell sind.

Methoden. Hierzu werden Zustrom, Zentrum und Abstrom des Schadensherdes im Hinblick auf Änderungen der Parameter Redoxpotenzial, Sauerstoff-, Nitrat-, Sulfat-, Eisen(II)-, Mangan(II)-, Hydrogencarbonat- und Calciumkonzentration untersucht. Die Ergebnisse werden sodann in einer Abfolge von lokalen Darstellungen in Form von Strahlendiagrammen verdeutlicht.

Ergebnisse und Schlussfolgerungen. Für den Abbau von Kohlenwasserstoffen werden Mikroorganismen immer den Elektronenakzeptor benutzen, bei dem sie durch die entsprechenden Redoxreaktionen den maximalen Energiegewinn erzielen. Das bedeutet, solange Sauerstoff verfügbar ist, wird dieser genutzt. Nach dessen Erschöpfung tritt Nitrat an seine Stelle, was über Nitrit zur Bildung von Stickstoff oder Ammonium führt. Als nächstes können Mangan(IV) und Eisen(III) Spezies, die nur gering wasserlöslich sind und sich hauptsächlich in der Festphase befinden, als Elektronenakzeptoren dienen. Dies führt zu löslichen Mangan(II) und Eisen(II) Verbindungen im Grundwasser. Bevor schließlich methanogene Bedingungen eintreten, wird Sulfat zum geeigneten Elektronenakzeptor, wodurch Schwefelwasserstoff gebildet wird.

Die unterschiedlichen Prozesse der Mineralisierung von Kohlenwasserstoffen führen zur Produktion von $\mathrm{CO}_{2}$ und damit zu einer Erhöhung von $\mathrm{HCO}_{3}^{-}$im Grundwasser, wodurch das KalkKohlensäure-Gleichgewicht verschoben wird. Dies bedeutet die Bildung von löslichem $\mathrm{Ca}\left(\mathrm{HCO}_{3}\right)_{2}$ aus unlöslichem $\mathrm{CaCO}_{3}$ und damit eine Erhöhung der Gehalte von $\mathrm{Ca}^{2+}$ im Grundwasser. Der Abbau der Kohlenwasserstoffe durch Mikroorganismen führt somit zu einer charakteristischen Redoxzonierung und zur Änderung sekundärer Parameter wie $\mathrm{Ca}^{2+}$ und $\mathrm{HCO}_{3}{ }^{-}$.

Empfehlungen und Ausblick. Verfolgt man die zeitlichen und räumlichen Veränderungen einiger charakteristischer Grundwasserparameter, bietet sich eine einfache Möglichkeit, das vorhandene Potenzial des mikrobiologischen Abbaus einer Grundwasserverunreinigung im Hinblick auf Natural Attenuation (NA)-Prozesse abzuschätzen.

Schlagwörter: Elektronenakzeptor; Grundwasser; Kalk-Kohlensäure-Gleichgewicht; mikrobieller Abbau; Mineralisierung; Mineralölkohlenwasserstoff-Verunreinigung; Natürliche Selbstreinigung; Redoxzonierung; Schadstofffahne

\section{Abstract \\ Determination of redox zones in a mineral-oil contaminated aquifer}

Goal and Scope. The goal of this study is the investigation and the grafic presentation of the characteristic redox zonation in a mineral oil contaminated aquifer which will be formed in the plume downstream of the contamination source: Methanogenic conditions, sulfate-reduction, $\mathrm{Fe}(\mathrm{III})$-reduction, $\mathrm{Mn}$ (IV)-reduction, nitrate-reduction, aerobic conditions. By that indications type and degree of microbial degradation which is the most important part in Natural Attenuation (NA) processes can be obtained easily.

Methods. Changes of the groundwater parameters $\mathrm{Eh}, \mathrm{O}_{2}, \mathrm{NO}_{3}^{-}$, $\mathrm{SO}_{4}{ }^{2-}, \mathrm{Fe}^{2+}, \mathrm{Mn}^{2+}, \mathrm{HCO}_{3}-, \mathrm{Ca}^{2+}$ will be measured upstream, downstream and also in the centre of the plume. The results will be presented in a sequence of special diagrams.

Results and Conclusion. When microbial degradation of hydrocarbons takes place, a microbial community will always use that electron acceptor from which it will gain a maximum of energy by the corresponding redox-reactions. This means as long as oxygen is available this will be used. After its depletion nitrate serves as electron acceptor leading via nitrite to the formation of nitrogen or ammonia. Manganese (IV) and Iron (III) species which are rather insoluble are mainly available from the soil-phase, can act as electron acceptor as next, leading to soluble Manganese(II) and Iron(II) compounds in groundwater. Finally before methanogenic conditions occur sulphate will become a suitable electron acceptor leading to the formation of hydrogen sulphide. All these processes of mineralization of the hydrocarbons will lead to the production of $\mathrm{CO}_{2}$ and as consequence to an increase of $\mathrm{HCO}_{3}^{-}$in groundwater changing the calcareous/carbonic acid-equilibrium. By that more soluble $\mathrm{Ca}\left(\mathrm{HCO}_{3}\right)_{2}$ is formed from insoluble $\mathrm{CaCO}_{3}$, so the concentration from $\mathrm{Ca}^{2+}$ will also increase. Thus, by the action of microorganisms, a typical redox-zonation and changes of other parameters will occur.

Recommendations and Perspective. To follow the changes in time and space of some characteristic groundwater parameters is a simple way to estimate the potential of microbial degradation in a contaminated aquifer considering Natural Attenuation (NA)-processes.

Keywords: Calcerous/carbonic acid-equilibrium; contamination plume; elecron acceptor; groundwater; microbial degradation; mineralization; mineral oil contamination; natural attenuation; redox zonation 


\section{Hintergrund und Zielsetzung}

Untergrundverunreinigungen durch Mineralöl und Mineralölprodukte sind in industrialisierten Ländern weit verbreitet. Die Tatsache, dass Mineralölkohlenwasserstoffe eine vergleichsweise gute Abbaubarkeit aufweisen, macht man sich in der Sanierungspraxis bereits im großem Umfang zunutze. Hier zählt die mikrobiologische Behandlung, insbesondere ex-situ, zu den bewährten Sanierungsverfahren. Zur Optimierung der Abbauprozesse werden häufig gezielt Nährstoffe und Elektronenakzeptoren zugegeben. Die Erfahrungen bei der Bearbeitung von Grundwasserverunreinigungen durch Mineralöl zeigen aber auch, dass in vielen Fällen im Abstrom des Schadensherdes auch ohne Einflussnahme beachtliche Abbauprozesse stattfinden. Die Reichweite der Belastungsfahne ist unter dieser Voraussetzung nur begrenzt und quasi-stationär. Sofern eine vollständige Mineralisierung der organischen Verbindungen stattfindet oder diese in Huminstoffe eingebaut werden, ist im weiteren Abstrom mit keiner Beeinträchtigung der Grundwasserqualität zu rechnen.

Vor diesem Hintergrund wird in den letzten Jahren immer mehr die Altlastenbearbeitung unter Einbeziehung des natürlichen Reinigungsvermögens, die in der angelsächsischen Literatur als 'Natural Attenuation' (NA) bezeichnet wird, diskutiert $[1,2]$. Eine Grundvoraussetzung für die praktikable Anwendbarkeit von NA ist jedoch der Nachweis mikrobiologischer Abbauprozesse. Der aerobe Abbau von Kohlenwasserstoffen ist schon an die hundert Jahre bekannt und galt lange als die einzige Abbaumöglichkeit. Die Kohlenwasserstoffverbindungen werden im Idealfall im Zuge der Mineralisierung bis zu den Endstufen $\mathrm{CO}_{2}$ und $\mathrm{H}_{2} \mathrm{O}$ abgebaut [3-5].

Es wurde lange Zeit angenommen, dass Kohlenwasserstoffe nur bei Anwesenheit von molekularem Sauerstoff abgebaut werden können. Der Sauerstoff ist notwendig um Hydroxylgruppen einzufügen und den Abbau zu starten. Erst in den letzten 10 bis 15 Jahren konnte nachgewiesen werden, dass spezielle Mikroorganismen in der Lage sind, Kohlenwasserstoffverbindungen unter verschiedenen anaeroben Bedingungen abbauen zu können [6]. Dabei fungieren entweder Nitrat [7-10], Sulfat [11-14], Eisen(III) $[15,16]$ oder Mangan(IV) [17] als Elektronenakzeptoren (Bildung von $\mathrm{CO}_{2}$ ) oder aber der Abbau findet unter methanogenen Bedingungen (Bildung von $\mathrm{CH}_{4}$ ) statt $[18,19]$, wobei Carbonat als Elektronenakzeptor genutzt wird. Verschiedene Faktoren können den mikrobiellen Abbau von Kohlenwasserstoffen aber auch limitieren.

Die Verfügbarkeit von Nährstoffen (z.B. Phosphor) und von geeigneten Elektronenakzeptoren, die Toxizität von Kontaminanten und ungünstige hydrochemische Bedingungen, wie z.B. zu hoher Salzgehalt, zu hohe oder zu niedrige $\mathrm{pH}$-Werte können sich auf mikrobielle Abbauprozesse negativ auswirken. Auch hat die Häufigkeit relevanter Mikroorganismen und die über Diffusion zur Verfügung stehenden Kohlenwasserstoffe einen Einfluss auf Abbauprozesse. Des weiteren spielt die Kinetik und die Energie der Aktivierungsreaktion zum Abbau und die Effizienz der nachfolgenden Reaktionen, die zu $\mathrm{CO}_{2}$ oder $\mathrm{CH}_{4}$ führen, eine Rolle [10].

Die in einem Aquifer auftretenden Mikroorganismen konkurrieren um vorhandene Ressourcen an Elektronendonatoren (z. B. organische Substrate) und Elektronenakzeptoren (z. B. Sauerstoff oder Nitrat).

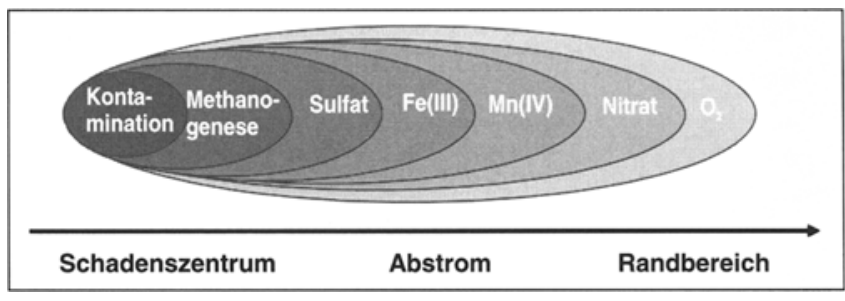

Abb. 1: Redoxzonierung in einer Schadstoffahne

Aus einem Pool von potentiellen Elektronenakzeptoren wird eine mikrobielle Gemeinschaft immer die aussuchen, die bei den entsprechenden Redoxreaktionen ein Maximum an Energie erbringen. Schließlich werden sich jeweils die Mikroorganismen durchsetzen, die an die gegebenen Bedingungen physiologisch am besten angepasst sind. Für die Redoxzonen im Aquifer mit einer organischen Verunreinigung bildet sich folgende Redoxzonierung im Abstrom der Kontamination aus: Methanogene Bedingungen - Sulfatreduktion - Eisen(III)-Reduktion - Mn(IV)-Reduktion - Nitratreduktion - aerobe Bedingungen [20]. Abb. 1 zeigt diese theoretische Redoxzonierung in Abstrom eines Schadenszentrums über die Fahrienlänge.

Viele Grundwasserverunreinigungen durch Mineralöl verfügen jedoch über ein unzureichendes Potenzial hinsichtlich der natürlichen Selbstreinigung, nicht etwa weil es an geeigneten Mikroorganismen fehlt, wohl aber an den Lebensbedingungen, die die Mikroorganismen zur Entfaltung ihrer vollen Aktivität benötigen [21]. Daher ist es wichtig, nicht nur die Art und Konzentration der Mineralölkohlenwasserstoffe im Aquifer zu kennen, sondern auch die mikrobiellen Aktivitäten und die vorhandenen Elektronenakzeptoren, die für die Stoffwechselleistung der Mikroorganismen von Bedeutung sind. Aus der Kenntnis der vorherrschenden Milieubedingungen, denen die Mikroorganismen ausgesetzt sind, lassen sich dann die nötigen Sanierungsmaßnahmen ableiten.

Ziel dieser Untersuchung ist es daher, die Schadstofffahne eines Altlaststandortes hinsichtlich des Redoxmilieus anhand primärer und sekundärer Parameteränderungen mit Hilfe der Standarduntersuchungsverfahren Redoxpotenzial, Sauerstoff-, Nitrat-, Sulfat-, Eisen(II)-, Mangan(II)-, Hydrogencarbonat- und Calciumkonzentration zu charakterisieren und die aktuelle Redoxsituation synoptisch deutlich zu machen. Anhand dieser Darstellungen können Hinweise auf Art und Ausmaß mikrobiologischer Abbauprozesse, die bei Anwendung von Verfahren wie z.B. Natural Attenuation (NA) essentiell sind, schnell und einfach erhalten werden.

\section{Methoden}

Die nachfolgend aufgeführten physikalisch-chemischen Untersuchungsergebnisse stammen aus einem zweijährigen Forschungsverbundvorhaben $\mathrm{zu}$ grundlegenden Untersuchungen der Rahmenbedingungen für Natural Attenuation, an dem die Fachhochschule Weihenstephan durch Charakterisierung eines Modellstandortes in Oberbayern (MKW-I BTEX-Schadensfall) beteiligt war.

Im März, Juni und Oktober 2002 wurden zur Erfassung der räumlichen und zeitlichen Verteilung der Schadstoffgehalte bzw. der Parameter, die auf mikrobiologische Abbauvorgänge 
Tabelle 1: Grundwasser-Untersuchungsverfahren

\begin{tabular}{l|l|l|l}
\hline Parameter & Untersuchungsort & Verfahrenshinweis & Methode \\
\hline Sauerstoff & Vorort & Elektrometrisch & EN 25814 \\
\hline Redoxpotenzial & Vorort & Elektrometrisch & DIN 38404 Teil 6 \\
\hline Nitrat & Labor & lonenchromatografie & EN ISO 10304-1 \\
\hline Sulfat & Labor & Ionenchromatografie & EN ISO 10304-1 \\
\hline Eisen(II) & Vorort & Fotometrisch & analog DIN 38406 Teil 1 \\
\hline Mangan(II) & Vorort & Fotometrisch & analog DIN 38406 Teil 2 \\
\hline Hydrogencarbonat & Vorort & Titrimetrisch & DIN 38409 Teil 7 \\
\hline Calcium & Labor & ICP-OES & EN ISO 11885 \\
\hline
\end{tabular}

schließen lassen, Grundwasserprobenahmen und Bodenluftmessungen durchgeführt. Teil des umfangreichen Untersuchungsprogrammes waren u.a. die in Tabelle $\mathbf{1}$ aufgeführten Untersuchungsverfahren.

Alle verwendeten Messgeräte wurden kalibriert und weitere geeignete Maßnahmen zur Analytischen Qualitätssicherung durchgeführt. Die Untersuchungsergebnisse wurden, sofern erforderlich, blindwert-korrigiert.

In der Bodenluft wurden Vorort die Parameter Methan, Schwefelwasserstoff, Kohlendioxid und Sauerstoff mittels IR-Gassensor bestimmt.

\section{Ergebnisse und Diskussion}

In Bodenluftproben aus dem Schadenszentrum konnten sowohl $\mathrm{CH}_{4}$ (4 bis $6 \mathrm{Vol}-\%$ ) und $\mathrm{H}_{2} \mathrm{~S}$ (bis ca. 1,4 ppm) als auch $\mathrm{CO}_{2}(2,6$ bis $6,9 \mathrm{Vol}-\%)$ bestimmt werden; die $\mathrm{O}_{2}$ Konzentrationen waren signifikant erniedrigt. In den Messstellen des Abstrombereiches konnten $\mathrm{CH}_{4}$ und $\mathrm{H}_{2} \mathrm{~S}$ nicht mehr nachgewiesen werden. $\mathrm{CO}_{2}$ in der Bodenluft war selbst in Messstellen des Randbereiches teilweise noch bis ca. 2 Vol$\%$ bestimmbar. Somit waren im Schadenszentrum methanogene Bedingungen eindeutig nachweisbar. Im Abstrom und im Randbereich jedoch wurden andere Elektronenakzeptoren als Carbonat für den mikrobiellen Abbau verwendet.
In den Ergebnistabellen 2-4 sind die Grundwasser-Analysenergebnisse einer Messstelle im Zustrom der Schadstoffquelle (GER 120), zweier Messstellen im Schadenszentrum (GER 342 und GER 118), zweier Messstellen im Abstrom (GER 199 und GER 166) sowie zweier Messstellen im Randbereich (GER 163 und GER 167) wiedergegeben.

Die Zustrom-Messstelle repräsentiert die Hintergrundsituation ohne Beeinflussung durch die Schadstoffquelle. Im Zentrum der Schadstoffquelle waren gelöster Sauerstoff sowie Nitrat im Grundwasser nicht mehr nachweisbar oder zumindest nahe der Bestimmungsgrenze; Redoxpotenzial sowie Sulfat-Konzentration waren deutlich niedriger als im Zustrom; Eisen(II)-, Calcium- sowie die Hydrogencarbonat-Konzentrationen nahmen im Zentrum deutlich im Vergleich zur Zustrom-Situation zu. Für Mangan(II) konnte in zwei Messkampagnen eine leichte Zunahme der Konzentrationen im Schadstoffzentrum festgestellt werden. Im Abstrom sowie im Randbereich der Schadstoffquelle näherten sich die Stoffkonzentrationen und Potentiale mehr und mehr der Hintergrundsituation.

Die Ergebnisse der Messkampagnen vom März und Juni 2002 sind weitestgehend vergleichbar (Tabelle 2 und 3). Dies entspricht auch den ähnlichen hydrogeologischen Verhältnisse am Standort während dieser Messkampagnen.

Tabelle 2: Messkampagne März 2002

\begin{tabular}{l|c|c|c|c|c|c|c|c}
\hline Messstelle & $\begin{array}{c}\text { Sauerstoff } \\
(\mathbf{m g} / \mathbf{L})\end{array}$ & $\begin{array}{c}\text { Redoxpotenzial } \\
(\mathbf{m V})\end{array}$ & $\begin{array}{c}\text { Nitrat } \\
(\mathbf{m g} / \mathbf{L})\end{array}$ & $\begin{array}{c}\text { Sulfat } \\
\mathbf{m g} / \mathbf{L})\end{array}$ & $\begin{array}{c}\mathbf{M n}(\mathbf{I I}) \\
\mathbf{m g} / \mathbf{L})\end{array}$ & $\begin{array}{c}\mathbf{F e}(\mathrm{II}) \\
(\mathbf{m g} / \mathbf{L})\end{array}$ & $\begin{array}{c}\mathbf{C a}^{\prime} \\
(\mathbf{m g} / \mathbf{L})\end{array}$ & $\begin{array}{c}\mathbf{H C O}_{3}^{-} \\
(\mathbf{m g} / \mathbf{L})\end{array}$ \\
\hline GER 120 (Zustrom) & 10 & 317 & 19,3 & 15,5 & $<0,5$ & $<0,1$ & 64,2 & 211 \\
\hline GER 342 (Zentrum) & 0 & -14 & $<0,1$ & 0,80 & $<0,5$ & 2,72 & 177 \\
\hline GER 118 (Zentrum) & 0 & -57 & $<0,1$ & 0,11 & $<0,5$ & 0,28 & 182 & 571 \\
\hline GER 199 (Abstrom) & 0 & 114 & 0,2 & 8,8 & $<0,5$ & 0,29 & 155 & 610 \\
\hline GER 166 (Abstrom) & 8,9 & 459 & 9,2 & 17,0 & $<0,5$ & $<0,1$ & 115 & 303 \\
\hline GER 163 (Randbereich) & 9,2 & 364 & 17,8 & 14,6 & $<0,5$ & $<0,1$ & 73,1 & 214 \\
\hline GER 167 (Randbereich) & 9,6 & 440 & 20,6 & 14,4 & $<0,5$ & $<0,1$ & 56,5 & 220 \\
\hline
\end{tabular}

Tabelle 3: Messkampagne Juni 2002

\begin{tabular}{l|c|c|c|c|c|c|c|c}
\hline Messstelle & $\begin{array}{c}\text { Sauerstoff } \\
(\mathbf{m g} / \mathbf{L})\end{array}$ & $\begin{array}{c}\text { Redoxpotenzial } \\
(\mathbf{m V})\end{array}$ & $\begin{array}{c}\text { Nitrat } \\
(\mathbf{m g} / \mathbf{L})\end{array}$ & $\begin{array}{c}\text { Sulfat } \\
(\mathbf{m g} / \mathbf{L})\end{array}$ & $\begin{array}{c}\text { Mn(II) } \\
(\mathbf{m g} / \mathbf{L})\end{array}$ & $\begin{array}{c}\mathbf{F e}(\mathrm{II}) \\
(\mathbf{m g} / \mathbf{L})\end{array}$ & $\begin{array}{c}\mathbf{C a}^{\prime} \\
(\mathbf{m g} / \mathbf{L})\end{array}$ & $\begin{array}{c}\mathbf{H C O}_{3}^{-} \\
(\mathbf{m g} / \mathbf{L})\end{array}$ \\
\hline GER 120 (Zustrom) & 10,6 & 395 & 19,9 & 11,5 & $<0,5$ & $<0,1$ & 69,1 & 220 \\
\hline GER 342 (Zentrum) & 1,9 & 19 & $<0,1$ & 0,22 & 1,1 & 2,47 & 161 & 537 \\
\hline GER 118 (Zentrum) & 0,4 & -64 & $<0,1$ & 0,30 & 0,64 & 2,10 & 135 & 497 \\
\hline GER 199 (Abstrom) & 2,0 & 136 & $<0,1$ & 5,4 & $<0,5$ & $<0,1$ & 144 & - \\
\hline GER 166 (Abstrom) & 4,0 & 203 & 10,0 & 13,3 & $<0,5$ & $<0,1$ & 101 & 232 \\
\hline GER 163 (Randbereich) & 9,8 & 383 & 16,0 & 12,4 & $<0,5$ & $<0,1$ & 70,5 & 238 \\
\hline GER 167 (Randbereich) & 10,1 & 387 & 19,2 & 13,3 & $<0,5$ & $<0,1$ & 63,9 & 214 \\
\hline
\end{tabular}


Tabelle 4: Messkampagne Oktober 2002

\begin{tabular}{l|c|c|c|c|c|c|c|c}
\hline Messstelle & $\begin{array}{c}\text { Sauerstoff } \\
(\mathbf{m g} / \mathbf{L})\end{array}$ & $\begin{array}{c}\text { Redoxpotenzial } \\
(\mathbf{m V})\end{array}$ & $\begin{array}{c}\text { Nitrat } \\
(\mathbf{m g} / \mathbf{L})\end{array}$ & $\begin{array}{c}\text { Sulfat } \\
(\mathbf{m g} / \mathbf{L})\end{array}$ & $\begin{array}{c}\text { Mn(II) } \\
(\mathbf{m g} / \mathbf{L})\end{array}$ & $\begin{array}{c}\mathbf{F e}(\mathrm{II}) \\
(\mathbf{m g} / \mathbf{L})\end{array}$ & $\begin{array}{c}\mathrm{Ca} \\
(\mathbf{m g} / \mathbf{L})\end{array}$ & $\begin{array}{c}\mathbf{H C O}{ }^{-} \\
(\mathbf{m g} / \mathbf{L})\end{array}$ \\
\hline GER 120 (Zustrom) & 8,9 & 393 & 14,6 & 8,8 & $<0,5$ & $<0,1$ & 80,8 & 305 \\
\hline GER 342 (Zentrum) & 0 & -70 & 0,35 & 3,7 & 0,54 & 2,80 & 138 & 506 \\
\hline GER 118 (Zentrum) & 0,6 & 150 & 0,7 & 4,4 & $<0,5$ & 0,96 & 143 & 500 \\
\hline GER 199 (Abstrom) & 1,7 & -6 & 0,2 & 2,0 & $<0,5$ & 0,33 & 135 & 464 \\
\hline GER 166 (Abstrom) & 1,4 & 385 & 4,5 & 8,4 & $<0,5$ & $<0,1$ & 107 & 403 \\
\hline GER 364 (Randbereich) & 8,0 & 356 & 12,8 & 8,9 & $<0,5$ & $<0,1$ & 75,9 & 317 \\
\hline GER 363 (Randbereich) & 8,8 & 313 & 13,9 & 8,4 & $<0,5$ & $<0,1$ & 75,0 & 287 \\
\hline
\end{tabular}

Die Ergebnisse der Messkampagne vom Oktober 2002 waren zwar in ihrer tendenziellen Aussage hinsichtlich Abnahme oder Zunahme von Stoffkonzentrationen oder Potentialen ebenfalls mit den vorhergehenden Ergebnissen vergleichbar, jedoch konnten im Oktober deutliche Verschiebungen beobachtet werden, die auf Variabilitäten von Reaktionszonen hinweisen (Tabelle 4). Diese sind auf geänderte hydrogeologische Verhältnisse am Standort zurückzuführen, da der Messkampagne mehrere Starkregenereignisse vorausgingen. Höhere Niederschlagsmengen führten im Aquifer zu Verdünnungseffekten und zu Verlagerungen des Schadstoffinventars.

Die Ergebnisse der Messkampagnen wurden in Form von Isoliniendarstellungen in Karten des Standortes übertragen. In dieser Darstellungsform kann die räumliche Ausbreitung der Schadstofffahne sowie deren zeitliche Variation gut erkannt werden [22]. In dieser Arbeit wird jedoch einer zusätzlichen Darstellungsform in Strahlendiagrammen der Vorzug gegeben (Abb. 2-4). Hierzu werden zur Darstellungsangleichung einige Parameter (und zwar Fe(II), Mn(II), Redoxpotenzial, Calcium und Hydrogencarbonat) normiert, indem diese Parameter wegen der sehr unterschiedlichen Konzentrationsniveaus mit Multiplikatoren bzw. Divisoren versehen werden. In einer Abfolge von lokalen Darstellungen in Form solcher Strahlendiagramme lässt sich dann die Änderung der Redoxzonierung entlang der Fahne vom Schadenszentrum zum Randbereich sehr gut überblicken, weil die $\mathrm{Zu}$ - oder Abnahme signifikanter Grundwasserparameter im Vergleich zur Zustrommessstelle erkennbar werden. In dieser Form wird ein aussagekräftigerer Eindruck über Art und Ausmaß von mikrobiologischen Abbauprozessen am Standort vermittelt.

Im Zuge von mikrobiologischen Abbauprozessen erfolgt im Schadenszentrum eine Abnahme der Parameter $\mathrm{O}_{2}$, Redox, Nitrat und Sulfat sowie eine Zunahme der Parameter Mangan(II), Eisen(II), Calcium und Hydrogencarbonat. Sofern im Strahlendiagramm die Parameter, die im Zuge von Abbauprozessen abnehmen, auf einer Seite des Strahlendiagramms (hier z.B. rechts) angeordnet werden und die Parameter, die im Zuge von Abbauprozessen zunehmen, auf der anderen Seite des Strahlendiagramms (hier z.B. links) dargestellt werden, wird der Umfang der Abbauprozesse durch das Maß der Auslenkung von der Messstelle, die die Zustromsituation repräsentiert, deutlich erkennbar.

Im Schadenszentrum sind die Parameteränderungen im Vergleich zur Zustromsituation (GER 120) maximal; die Flä- chen der Messstellen im Schadenszentrum GER 342 und GER 118, die durch Abtragung der Konzentrationen auf dem Strahlendiagramm aufgespannt werden, sind räumlich vollständig getrennt von der Fläche der Zustrommessstelle (Bild a 'Schadenszentrum' der Messkampagne März 2002 in Abb. 2).

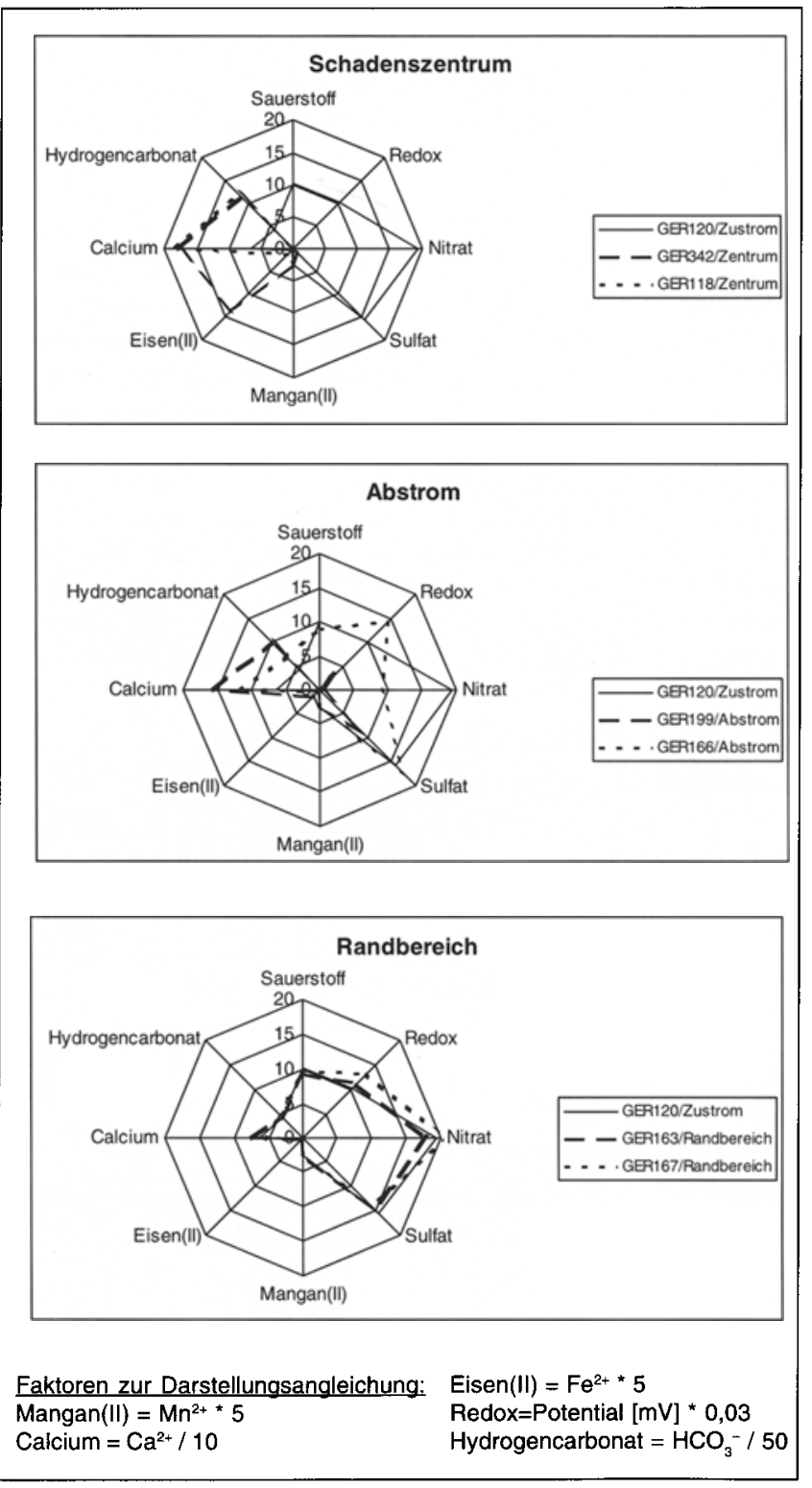

Abb. 2: Strahlendiagramm der Redoxzonierung Messkampagne März 2002 
Im Abstrombereich wandern die im Strahlendiagramm aufgespannten Flächen der Messstellen GER 199 und GER 166 mit zunehmender Entfernung vom Schadenszentrum weiter nach rechts und nähern sich der Zustromsituation (GER 120) immer weiter an (Bild b 'Abstrom' der Messkampagne März 2002 in Abb. 2).

Im Randbereich sind die im Strahlendiagramm aufgespannten Flächen der Messstellen GER 163 und GER 167 nahezu identisch mit der Fläche Zustrommessstelle GER 120, d.h. eine Redoxzonierung ist nicht mehr nachweisbar, die ursprünglichen Parameterkonzentrationen, wie sie im Zustrom erhalten worden sind, sind wieder erreicht. Daraus kann geschlossen werden, dass keine die Kontamination betreffenden mikrobiologischen Abbauprozesse mehr stattfinden (Bild c 'Randbereich' der Messkampagne März 2002 in Abb. 2). Vergleichbare Redoxzonierungen wurden auch in den Messkampagnen Juni 2002 und Oktober 2002 erhalten (Abb. 3 und 4).

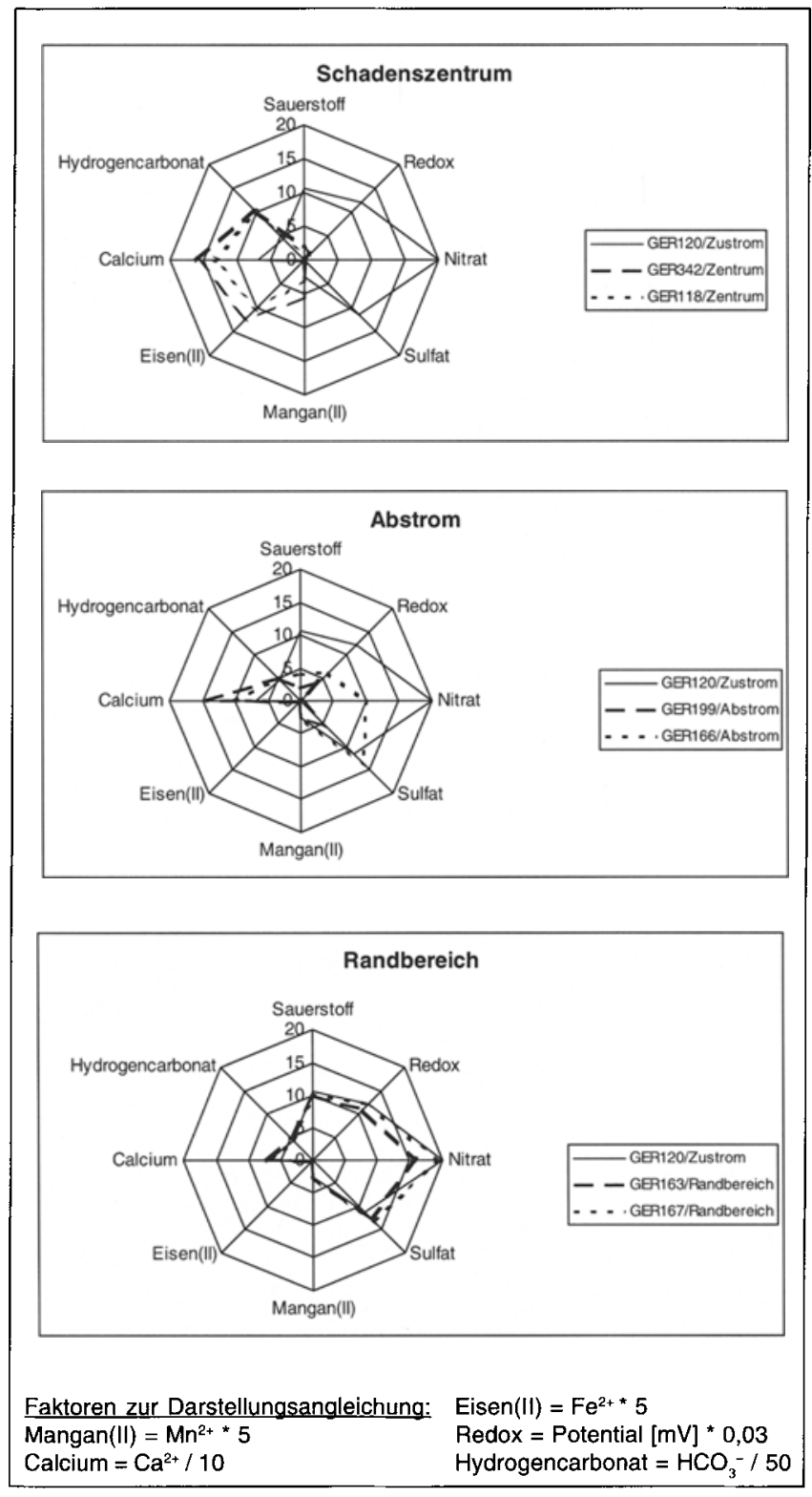

Abb. 3: Strahlendiagramm der Redoxzonierung Messkampagne Juni 2002
Redoxzonierungen treten im Allgemeinen nicht nur horizontal, sondern auch vertikal auf. Daher sind normalerweise auch tiefenhorizontierte Probenahmen notwendig. Diese sind jedoch für den hier untersuchten Standort entbehrlich, da hier die Grundwassermächtigkeit mit 1-2 m als gering einzustufen ist. Weitere Faktoren, die bei der Bestimmung von Redoxzonen Beachtung finden sollten, sind geochemische Prozesse in Form von Ausfällungen. Bei Anwesenheit von Sulfid können Eisen(II) und Mangan(II) als Sulfide ausfallen und sind somit im Grundwasser nicht mehr nachzuweisen. Ebenso können redox-sensitive Parameter aus der aktiven Zone transportiert werden oder die verschiedenen Redoxzonen überlappen sich. Auch die Erscheinungsform der festen Bodenphase darf nicht vernachlässigt werden, da sich besonders reduziertes Eisen auch an Bodenphasen unterschiedlich stark anlagern kann [23].

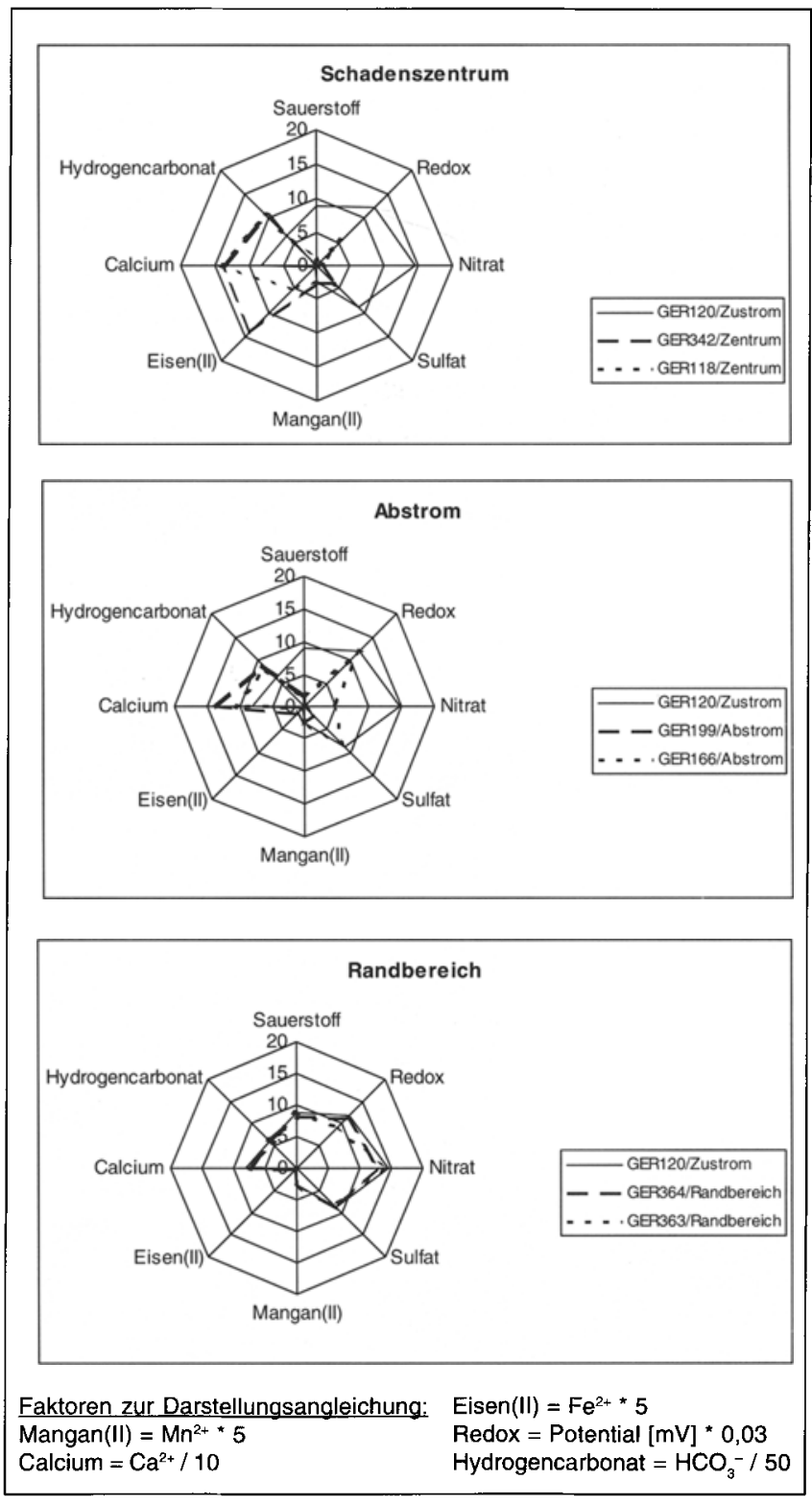

Abb. 4: Strahlendiagramm der Redoxzonierung Messkampagne Oktober 2002 


\section{Schlussfolgerungen}

Im Zuge des aeroben mikrobiologischen Abbaus von organischen Schadstoffen wird zunächst im Grundwasser gelöster molekularer Sauerstoff gezehrt. Wenn dieser aufgebraucht ist, beginnen die Mikroorganismen den gebundenen Sauerstoff des Elektronenakzeptors Nitrat für den Abbau der organischen Schadstoffe zu $\mathrm{CO}_{2}$ zu verwenden. Dabei wird Nitrat über Nitrit zu Stickstoff oder Ammoniak reduziert. Eisen(III) und $\mathrm{Mn}(\mathrm{IV})$-Spezies, welche überwiegend als schwerlösliche Verbindungen in der festen Bodenphase vorhanden sind, wirken ebenfalls als Elektronenakzeptoren und bilden an der Grenzfläche von fester und flüssiger Phase leichtlösliche Eisen(II)- und $\mathrm{Mn}(\mathrm{II})$-Verbindungen, die sich in Folge im Grundwasser nachweisen lassen. Schließlich dient Sulfat als Elektronenakzeptor wobei Sulfat zu Schwefelwasserstoff reduziert wird. Der durch die Abbauprozesse verursachte massive Eintrag von $\mathrm{CO}_{2}$ ins Grundwasser stört das lokale KalkKohlensäure-Gleichgewicht. Die Folge davon ist, dass die Konzentration an Hydrogencarbonat im Grundwasserleiter stark ansteigt und damit auch vermehrt schwerlösliches Calciumcarbonat aus der festen Bodenphase in leichtlösliches Calciumhydrogencarbonat überführt wird. Daher steigt auch die Konzentration an Calcium im Aquifer beträchtlich an. Bei weitergehendem mikrobiologischem Abbau stellen sich im Aquifer methanogene Bedingungen ein, die durch ein sehr niedriges Redoxpotenzial des Grundwasserleiters geprägt sind. In diesem Milieu werden organische Schadstoffen schließlich anaerob unter Zuhilfenahme von Carbonat als Elektronenakzeptor oder aber auch in Gärungsprozessen abgebaut.

Am untersuchten Standort ist eine typische Abfolge von Redoxzonen mit methanogenen, sulfatreduzierenden, eisen(III)reduzierenden und nitratreduzierenden Bedingungen, die auf natürliche Abbauprozesse hindeuten, gut zu erkennen.

Die stark sandigen Kiese am untersuchten Standort sind eisen- und manganarm, aufgrund dessen steht im Bodenkörper nicht genügend Eisen und Mangan zur Nachlieferung von Eisen(II) und $\mathrm{Mn}$ (II) ins Grundwasser zur Verfügung. Daher ist die Konzentration an Eisen(II) im Schadenszentrum nicht sonderlich hoch; Mangan(II) ist im kontaminierten Bereich sogar nur in Spuren nachweisbar.

\section{Empfehlungen und Ausblick}

Anhand der Parameteränderungen von Sauerstoff, Redoxpotenzial, Nitrat, Eisen(II), Mangan(II) und Sulfat im kontaminierten Bereich und im Abstrom lässt sich die Redoxzonierung darstellen. Als sekundäre Folgereaktionen im Zuge der Schadstoffmineralisierung $\mathrm{zu} \mathrm{CO}_{2}$ ändern sich auch Parameter wie Calcium und Hydrogencarbonat. Über diese Darstellung erhält man zusätzlich zu den Schadstoffgehalten und den mikrobiologischen Untersuchungen Hinweise auf die räumliche und zeitliche Ausdehnung der natürlichen Abbauprozesse.

Die Ermittlung der Redoxzonierung eines mineralölbelasteten Aquifers ist damit wichtige Voraussetzung zur Beurteilung, ob eine Altlastenbearbeitung unter Verwendung von NA zum Erfolg führen kann. Darüber hinaus dient die Redoxzonierung als entscheidendes Beobachtungskriterium für die räumliche und zeitliche Variabilität der Schadstoffquelle bei der Langzeitüberwachung.
Danksagung. Die Autoren danken dem Bayerischen Staatsministerium für Landesentwicklung und Umweltfragen für die finanzielle Unterstützung im Rahmen des Bayerischen Forschungsverbundvorhabens 'Altlasten. bewältigung unter Einbeziehung des natürlichen Reinigungsvermögens'.

\section{Literatur}

[1] Teutsch G, Grathwohl P (1999): Literaturstudie zum natürlichen Rückhalt/Abbau von Schadstoffen im Grundwasser. Nachdruck von Band 35/97 aus der Reihe Texte und Berichte zur Altlastenbearbeitung der Landesanstalt für Umweltschutz Baden-Württemberg

2] Rügner H, Grathwohl P, Teutsch G, Kohler W (2001): Natural Attenuation organischer Schadstoffe im Grundwasser. Schriftenreihe altlastenforum Baden-Württemberg e.V., Heft 5

[3] Nyer E, Boettcher G, Morelle B (1991): Using the properties of organic compounds to help design a treatment system. Ground Water Monitoring Review 11(4) 81-87

44] Mackenbrock U, Kopp-Holtwiesche B, Blank W (1994): Zur biologischen Abbaubarkeit von Industriechemikalien. TerraTech 4/94, 41-51

[5] Fritsche W (1998): Umweltmikrobiologie. Grundlagen und Anwendungen, Gustav Fischer Verlag Jena

[6] Heider J, Fuchs G (1997): Anaerobic metabolism of aromatic compounds. Eur J Biochem 243, 577-596

[7] Song B, Häggblom MM, Zhou J, Tiedje JM, Palleroni NJ (1999): Taxonomic characterization of denitrifying bacteria that degrade aromatic compounds and description of Azoarcus toluvorans sp. Nov, and Azoarcus toluclasticus sp. Nov. Int J Syst Bacteriol 49, 1129-1140

[8] Burland SB, Edwards EA (1999): Anaerobic benzene biodegradation linked to nitrate reduction. Appl Environ Microbiol 65, 529-533

[9] Ehrenreich P, Behrends A, Harder J, Widdel F (2000): Anaerobic oxidarion of alkanes by newly isolated denitrifying bacteria. Arch Microbiol 173, 58-64

[10] Spormann AM, Widdel F (2000): Metabolism of alkylbenzenes, alkanes and other hydrocarbons in anaerobic bacteria; Biodegradation 11, 85-105

[11] Lovley DR (1995): Bioremediation of organic and metal contaminants with dissimilatory metal reduction. J Ind Microbiol 14, 85-93

[12] Beller HR, Spormann AM, Sharma PK, Cole JR, Rheinhard M (1996): Isolation and characterization of a novel toluene-degrading sulphatereducing bacterium. Appl Environ Microbiol 62, 1188-1196

[13] Harms G, Zengler K, Rabus R, Aeckersberg F, Minz D, Rosello-Mora R, Widdel F (1999): Anaerobic oxidation of o-xylene, m-xylene and homologous alkylbenzenes by new types of sulphate-reducing bacteria. Appl Environ Microbiol 65, 999-1004

[14] So CM, Young LY (1999): Isolation and characterization of a sulphatereducing bacterium that anaeobically degrades alkanes. Appl Environ Microbiol 65, 2969-2976

[15] Rooney-Varga JN, Anderson RT, Fraga IL, Ringelber D, Lovley DR (1999): Microbial communities associated with anaerobic benzene degradation in a petroleum-contaminated aquifer. Appl Environ Microbiol 65, 3056-3063

[16] Anderson RT, Lovley DR (1999): Naphthalene and benzene degradation under $\mathrm{Fe}(\mathrm{III})$-reducing conditions in petroleum-contaminated aquifers. Environ Sci Technol 34, 2261-2266

[17] Langenhoff AAM, Nijenhuis I, Tan NCG, Briglia M, Zehnder AJB, Schraa G (1997): Characterization of a manganese-reducing toluenedegrading enrichment culture. FEMS Microbiol Ecol 24,113-125

[18] Anderson RT, Lovley DR (2000): Hexadecane decay by methanogenesis. Nature 404, 722-723

[19] Beller HR, Edwards EA (2000): Anaerobic toluene activation by benzylsuccinate synthase in a highly enrichment methanogenic culture. Appl Environ Microbiol 66, 5503-5505

[20] Lovley DR, Woodward JC, Chapelle FH (1994): Use of dissolved $\mathrm{H}_{2}$ concentrations to determine distribution of microbially catalysed redox reactions in anoxic groundwater. Environ Sci Technol 28, 1025-1210

[21] Krist D (2002): Adaptierung eines DIN-genormten Prüfverfahrens zur Bestimmung von Mineralölkohlenwasserstoffen in Wasserproben mittels GC/FID, Diplomarbeit Fachhochschule Weihenstephan, Fachbereich Unweltsicherung

[22] Kiesel M, Harlacher C, Pyka W, Hartmann R, Huth R (2003): Forschungsverbundvorhaben 'Nachhaltige Altlastenbewältigung unter Einbeziehung des natürlichen Reinigungsvermögens', Teilthema 'Felduntersuchungen zur Quantifizierung und Bewertung der natürlichen Abbauvorgänge im Grundwasser bei Verunreinigungen durch Mineralölkohlenwasserstoffe am Beispiel des Standorts Geretsried'. Fachhochschule Weihenstephan, Fachbereich Umweltsicherung

[23] Christensen TH, Bjerg PL, Banwart SA, Jakobsen R, Heron G, 2000 , Characterization of redox conditions in groundwater contaminant plumes. J Contam Hydrol 45, 165-241

Eingegangen: 15. Dezember 2003

Akzeptiert: 19. August 2004

OnlineFirst: 20. August 2004 